\title{
Sequela de cirurgia para reconstrução mandibular após ressecção de ameloblastoma
}

Post-surgical sequela of mandibular reconstruction after resection of ameloblastoma

Sequela de cirugía para reconstrucción mandibular después de la resección de ameloblastoma

\author{
Allancardi dos Santos SIQUEIRA ${ }^{1}$ \\ Luiz Henrique Soares TORRES ${ }^{2}$ \\ Jiordanne Araújo DINIZ ${ }^{3}$ \\ Éwerton Daniel Rocha RODRIGUES ${ }^{3}$ \\ Caio Pimenteira UCHOA ${ }^{4}$ \\ Valfrido Antônio PEREIRA FILHO² \\ Ana Cláudia Amorim GOMES ${ }^{4}$ \\ Emanuel Dias de OLIVEIRA E SILVA
}

${ }^{I}$ Cirurgião Bucomaxilofaial pelo Hospital Universitário Oswaldo Cruz, Universidade de Pernambuco, UPE, 50100-130 Recife - PE, Brasil

${ }^{2}$ Programa de Pós-graduação em Ciências Odontológicas, Departamento de Diagnóstico e Cirurgia, Universidade Estadual Paulista “Júlio de Mesquita Filho" (UNESP) Faculdade de Odontologia de Araraquara, 14801-903, Araraquara - SP, Brasil

${ }^{3}$ Programa de Pós-graduação em Cirurgia e Traumatologia Bucomaxilofacial, Hospital Universitário Oswaldo Cruz, UPE Universidade de Pernambuco, 50100-130 Recife - PE, Brasil

${ }^{4}$ Departamento de Cirurgia e Traumatologia Bucomaxilofacial, Hospital Universitário Oswaldo Cruz, UPE Universidade de Pernambuco, 50100-130 Recife - PE, Brasil

\section{Resumo}

Os defeitos mandibulares devido à ressecção de lesão óssea interferem a harmonia e estética facial comprometendo a qualidade de vida dos pacientes. Grandes defeitos exigem planejamento minucioso, principalmente quando lançamos mão de enxertos e placas de reconstrução, evitando assim resultados insatisfatórios ou até mesmo sequelas. Apesar de algumas classificações dos defeitos mandibulares vêm sendo discutidas ao longo dos anos, ainda não há um protocolo definido para reconstrução mandibular. O uso de prototipagem na Cirurgia Bucomaxilofacial tem se tornado cada vez mais frequente; a precisão da reconstrução, diminuição do tempo de cirurgia reflete em recuperação mais rápida do paciente. Mesmo com a ferramenta da prototipagem, grandes lesões e perdas ósseas permanecem como grande desafio ao cirurgião. O objetivo deste trabalho é relatar um caso clínico de reconstrução mandibular no qual houve a necessidade de ser tratado com remoção de placa de reconstrução e nova reabilitação cirúrgica do paciente.

Descritores: Mandíbula; Reconstrução Mandibular; Modelos Biológicos.

\section{Abstract}

Mandibular defects due to resection of bone lesions interfere with harmony and facial aesthetics, compromising patients' quality of life. Large defects require careful planning, especially when we use grafts and rebuilding plates, thus avoiding unsatisfactory results or even sequelae. Although some classifications of mandibular defects have been discussed over the years, there is still no definite protocol for mandibular reconstruction. The use of prototyping in Buccomaxillofacial Surgery has become increasingly frequent; the accuracy of the reconstruction, decreased time of surgery reflected in the patient's faster recovery. Even with the prototyping tool, large lesions and bone losses remain a major challenge to the surgeon. The objective of this work is to report a clinical case of mandibular reconstruction in which there was a need to be treated with removal of plaque from reconstruction and new surgical rehabilitation of the patient.

Descriptors: Mandible; Mandibular Reconstruction; Biological Models.

\section{Resumen}

Los defectos mandibulares debido a la resección de lesión ósea interfieren la armonía y estética facial comprometiendo la calidad de vida de los pacientes. Los grandes defectos requieren una planificación minuciosa, principalmente cuando lanzamos mano de injertos y placas de reconstrucción, evitando así resultados insatisfactorios o incluso secuelas. A pesar de algunas clasificaciones de los defectos mandibulares vienen siendo discutidas a lo largo de los años, todavía no hay un protocolo definido para la reconstrucción mandibular. El uso de prototipado en la Cirurgía Bucoaxilofacial se ha vuelto cada vez más frecuente; la precisión de la reconstrucción, la disminución del tiempo de cirugía refleja la recuperación más rápida del paciente. Incluso con la herramienta del prototipado, grandes lesiones y pérdidas óseas permanecen como gran desafío al cirujano. El objetivo de este trabajo es relatar un caso clínico de reconstrucción mandibular en el cual hubo la necesidad de ser tratado con remoción de placa de reconstrucción y nueva rehabilitación quirúrgica del paciente.

Descriptores: Mandíbula; Reconstrucción Mandibular; Modelos Biológicos.

\section{INTRODUÇÃO}

As reconstruções mandibulares e da cavidade bucal têm por objetivo dar suporte às estruturas de tecido mole e duro da região maxilofacial, sendo fundamental para o desempenho das suas funções biomecânicas e fisiológicas, além de manter o contorno facial e a estética. Defeitos bucomaxilofaciais podem levar a graves consequências funcionais e psicológicas ao paciente. A reconstrução mandibular é sempre necessária após ressecção parcial ou total da mandíbula, sendo desafiadora mesmo para cirurgiões experientes. A reconstrução primária de defeitos mandibulares é geralmente realizada imediatamente à ressecção de uma lesão. Em contrapartida, a secundária apresenta um grande desafio devido à presença de cicatrizes e contratura de tecido fiboso ${ }^{1,2}$. No entanto, atualmente, tem-se em mãos métodos coadjuvantes que auxiliam no planejamento de grandes reconstruções maxilofaciais.

$\mathrm{O}$ uso de prototipagem em cirurgia reconstrutora maxilofacial tem se tornado cada vez mais frequente. A compreensão dos detalhes anatômicos com alta qualidade, simulação de procedimentos cirúrgicos, produção e adaptação de biomateriais (placas, parafusos, próteses), precisão da reconstrução e diminuição do tempo cirúrgico, refletem em menor tempo de internação e recuperação mais rápida do paciente. Citam-se ainda redução de potenciais complicações pós-operatórias e melhor orientação/discussão do planejamento com o paciente ${ }^{3}$. O objetivo deste trabalho é relatar um caso clínico de reconstrução mandibular no qual houve necessidade de remoção da placa de reconstrução e nova reabilitação cirúrgica do paciente. 


\section{CASO CLÍNICO}

Paciente melanoderma, sexo masculino, 54 anos de idade, procurou o serviço de Cirurgia e Traumatologia Buco-Maxilo-Facial do Hospital Universitário Oswaldo Cruz (CTBMF/HUOC/UPE) em 2012 com uma lesão óssea expansiva em região de corpo mandibular à direita e, na oportunidade, foi realizada biópsia incisional e obtido diagnóstico de ameloblastoma. Estabeleceu-se um plano de tratamento conservador para a lesão, no entanto, o paciente não retornou ao serviço para acompanhamento. O mesmo retornou ao serviço de CTBMF do HUOC em 2017, após ter se submetido a uma ressecção parcial de mandíbula envolvendo todo o corpo mandibular à direita e parte da sínfise e parassínfise mandibular à esquerda com reconstrução imediata através de placa de reconstrução do sistema 2.4 e enxerto livre de ilíaco. Três anos após a intervenção o paciente evoluiu com reabsorção do enxerto, sequela estética e funcional.

Ao exame físico observa-se aspecto prognata pelo tamanho inadequado da placa de reconstrução, assimetria do terço inferior da face e eversão do lábio inferior (Figura 1A, B e C). O exame físico intrabucal mostra apagamento do fundo de vestíbulo mandibular, uma camada delgada de mucosa recobrindo a placa de reconstrução, sem recobrimento muscular aparente, além de comprometimento da mobilidade da língua. $\mathrm{O}$ exame de imagem evidencia placa de reconstrução com tamanho, modelagem e local de fixação inadequado (Figura 2A e B).

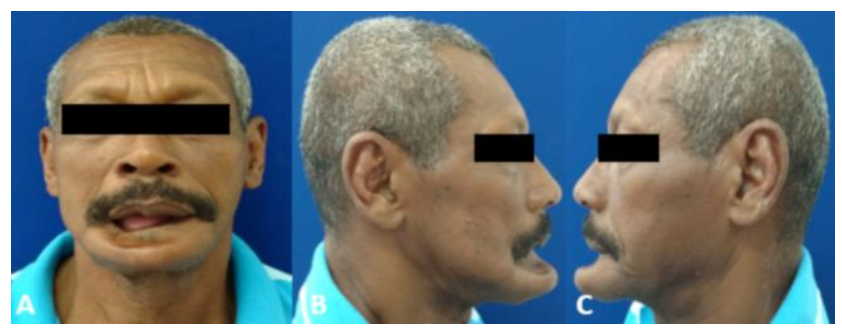

Figura1: Aspecto clínico do paciente exibindo prognatia pelo tamanho inadequado da placa de reconstrução, assimetria do terço inferior da face e eversão do lábio inferior.

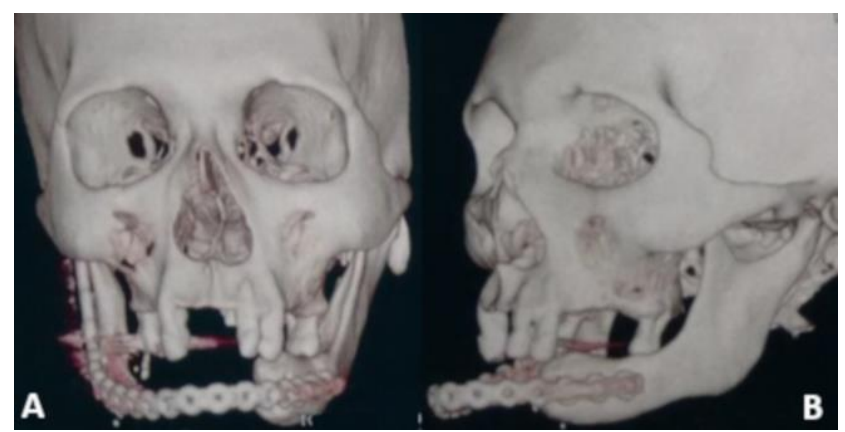

Figura 2: Exame de imagem evidenciando placa de reconstrução com tamanho, modelagem e local de fixação inadequado.

O novo planejamento cirúrgico visou substituição da placa por outra que atendesse aos princípios biomecânicos e estéticos aceitáveis para a reabilitação do paciente. A nova placa foi moldada com auxílio de prototipagem e planejamento com ceroplastia e "template" (Figura 3A, B e C).

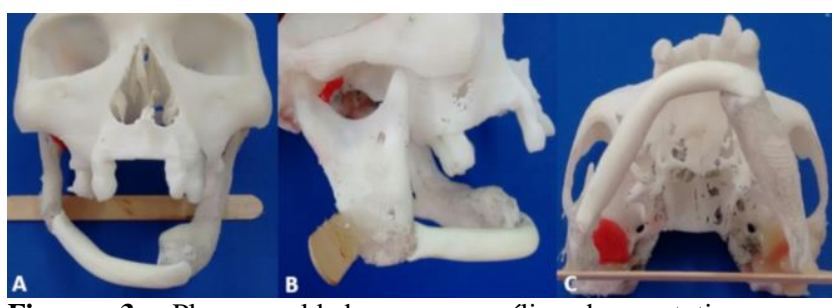

Figura 3: Placa moldada com auxílio de prototipagem e planejamento com ceroplastia e "template".

O paciente foi submetido à cirurgia sob anestesia geral sendo realizada incisão vestibular mandibular sob a região da placa e remoção da mesma. A nova placa com contorno adequado foi instalada e coberta por camada de tecido muscular e mucosa. O mesmo segue de acompanhamento de dois anos, apresentando resultado estético e funcional satisfatório (Figura 4A, B e C).

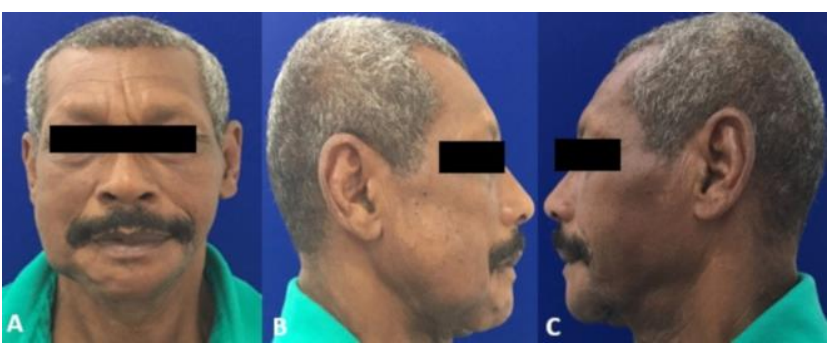

Figura 4: Resultado estético e funcional satisfatório. Pós-operatório de 2 anos.

\section{DISCUSSÃO}

A reconstrução imediata após uma ressecção em bloco com margens de segurança é a melhor alternativa para tratar ameloblastomas, uma vez que promove remoção total da lesão e tratamento cosmético e funcional no mesmo tempo cirúrgico ${ }^{4}$.

A ressecção parcial da mandíbula, seja devido à lesão ou por outros fatores, afeta diretamente a qualidade de vida dos pacientes ${ }^{5}$. A reconstrução mandibular é sempre necessária após ressecção óssea parcial e defeito de continuidade ${ }^{6}$. As alterações estéticas e funcionais vêm sendo minimizadas pelo uso de placas reconstrutivas modeladas previamente em protótipos ${ }^{1}$.

Mesmo com a melhoria contínua dos materiais, surgimento de novas tecnologias e técnicas cirúrgicas, o tratamento dessas grandes lesões e perdas ósseas permanece como grande desafio ao cirurgião ${ }^{5}$. Ao realizar a reconstrução mandibular, a restauração da continuidade óssea por si só não deve ser considerada a medida do sucesso: aspectos anatômicos e funcionais como mastigação, deglutição, fala e competências labiais devem ser objetos de análise, além da unidade estética como perfil e contorno facial ${ }^{7}$.

Neste relato o paciente retornou ao serviço após cinco anos da primeira consulta e três anos após intervenção cirúrgica, em outra unidade hospitalar, com reabsorção do enxerto livre de crista ilíaca, além 
de sequela estética e funcional.

Um dos princípios básicos da cirurgia reconstrutiva é manter o tecido ósseo remanescente com as mesmas relações anatômicas anteriores à ressecção da lesão, proporcionando a reconstrução com tecido duro e mole. Fatores fundamentais devem ser considerados quando se trata de propor uma reconstrução, dentre os quais o tamanho e a posição do defeito, a qualidade dos tecidos restantes, vascularização e condição geral do paciente. O resultado final é mais influenciado pela reconstrução do tecido mole do que mesmo pela reconstrução óssea $^{8}$. Nota-se no presente caso que os princípios básicos de reconstrução mandibular não foram obedecidos no primeiro tempo cirúrgico. $\mathrm{O}$ paciente apresentava aspecto prognata pelo tamanho inadequado da placa de reconstrução, eversão do lábio inferior, apagamento do fundo de vestíbulo mandibular, além de uma camada delgada de mucosa recobrindo a placa de reconstrução, sem recobrimento muscular aparente.

Uma das dificuldades quanto ao uso das placas de reconstrução, principalmente nos casos em que o defeito ressectivo envolve região de sínfise mandibular, é restabelecer um contorno facial aceitável devido à dificuldade de se promover uma perfeita adaptação da placa por conta da sua rigidez e do contorno mandibular ${ }^{1}$. O uso de prototipagem em cirurgia reconstrutora maxilofacial oferece possibilidade de discussão e planejamento, completo entendimento da anatomia óssea antes da cirurgia, estimar o comprimento da placa de reconstrução, adaptá-la e selecionar o número e o tamanho dos parafusos a serem usados, reduzindo assim o tempo cirúrgico e os riscos de danos a estruturas nobres como nervo, além de facilitar a restauração do contorno facial pela modelagem prévia da placa de reconstrução, podendo ser associada ou não a enxerto ósseo ${ }^{3,9}$.

A técnica de Fariña et al. ${ }^{9}$ é uma das mais usadas para modelagem de placas de reconstrução mandibular. Através de guias de resina acrílica, com impressões das superfícies oclusais e da placa de titânio, permite a replicação precisa de sua posição em o remanescente mandibular. Neste trabalho o defeito causado pela ressecção da lesão se estendia por todo corpo mandibular à direita e parte da sínfise e parassínfise mandibular à esquerda e foi necessário modelo prototipado para modelagem previa da placa de reconstrução. $\mathrm{O}$ paciente apresentava edentulismo total na mandíbula e parcial na maxila, promovendo uma oclusão instável; não permitindo assim a confecção de guias como preconizado por Fariña et al. ${ }^{9}$ Sendo assim, a nova placa foi moldada com auxílio de prototipagem e planejamento com ceroplastia e "template" levando em consideração a posição dos condilos mandibulares centralizados na cavidade glenóide
Segmentos mandibulares maiores que cinco centímetros tratados com enxertos ósseos livres tendem a ter uma maior taxa de complicações pósoperatórias ${ }^{10}$. Neste caso a escolha do enxerto livre de crista ilíaca não obedeceu aos princípios de reconstrução da mandíbula, levando assim ao insucesso. Os enxertos ósseos autógenos microvascularizados de fíbula com tecido muscular aderido são uma modalidade de tratamento confiável para a reconstrução de defeitos ósseos mandibulares, tendo menor taxa de reabsorção e falha; devendo ser a primeira escolha, associado a placas de reconstrução, para a maioria dos casos de reconstrução mandibular. No entanto é fundamental que a modalidade de tratamento para reconstrução mandibular seja individualizada para cada paciente ${ }^{7}$. Embora as técnicas reconstrutivas resultem em melhora significativa para os pacientes, desvantagens consideráveis ainda permanecem sendo que a reabilitação total do caso nem sempre é alcançada ${ }^{5}$. No caso em questão o paciente não aceitou passar por nova reconstrução com enxerto, sendo submetido apenas a remoção da placa mal adaptada e instalação de outra com melhor contorno anatômico, resolvendo sua queixa principal.

A reconstrução secundária é desafiadora devido à presença de cicatrizes e contratura de tecido fibroso, dificultando o recobrimento da placa de reconstrução com tecido muscular e mucosa bucal; as estratégias cirúrgicas para a reconstrução dos defeitos ósseos mandibulares muitas vezes variam de acordo com a localização e o tamanho dos defeitos ${ }^{2}$. Uma classificação e compreensão detalhada desses defeitos ajuda o cirurgião a planejar a reconstrução.

Alguns sistemas de classificação dos defeitos bucomaxilofaciais, tanto de componente ósseo quanto de tecido mole, vêm sendo discutido ao longo dos anos. Desde que a classificação de defeitos mandibulares de Pavlov em 1974 foi publicada, outros sistemas de classificação adicionais foram propostos. No entanto nenhuma dessas classificações é usada universalmente, e muitos trabalhos apenas descrevem os defeitos, sem propor métodos de reconstrução. Brown et al. ${ }^{11}$ em 2016 propôs uma classificação com objetivo de prever morbidade e orientar as reconstruções; Classe I (ângulo), Classe Ic (ângulo e côndilo), Classe II (ângulo e região de canino), Classe IIc (ângulo, região de canino e côndilo), Classe III (ambas regiões de caninos), Classe IV (ambas regiões de caninos e pelo menos um ângulo), Classe IVc (ambas regiões de caninos e pelo menos um côndilo).

$\mathrm{O}$ presente caso pertence à classificação Classe II de Brown et al. ${ }^{11}$ tendo indicação de reconstrução com crista ilíaca e placa de reconstrução, no entanto no primeiro tempo cirúrgico, outros princípios de reconstrução mandibular como a manutenção do osso 
remanescente com as mesmas relações anatômicas anteriores à ressecção da lesão e modelagem anatômica da placa de reconstrução, não foram obedecidos o que levou a resultado insatisfatório e sequela.

Apesar da classificação de Urken et al. ${ }^{12}$ em 1991 ser puramente descritiva e não prever morbidade ou sugerir um método de reconstrução óssea, é importante para classificar os defeitos do tecido mole. Os defeitos da mucosa são classificados como labiais e bucais, já os defeitos do assoalho bucal são classificado como anterior ou lateral e baseado na necessidade ou não de enxerto de tecido mole para evitar a obliteração do fundo de vestíbulo e preservar a mobilidade da língua.

Um defeito ósseo mandibular associado a um defeito de tecido mole clinicamente significativo que envolve estruturas complexas da cavidade bucal como assoalho e fundo de vestíbulo compromete diretamente a reconstrução mandibular. Dificilmente enxerto ósseo livre permitirá uma reabilitação e reconstrução bem sucedida nesse defeito, visto que o tecido mole não é suficientemente flexível ou tem um volume ideal para permitir a reconstrução adequada do defeito ${ }^{13}$. Afirmação que corrobora com Farina et al. ${ }^{9}$ ao relatar que o fator crítico quanto ao prognóstico da reconstrução é ter tecido mole de boa qualidade para recobrir o enxerto e/ou placa de reconstrução.

Wei et al. ${ }^{14}$ analisaram 80 pacientes com defeitos mandibulares, sendo 67 defeitos do corpo mandibular. Os casos foram reconstruídos com placa de reconstrução e tecido mole. Os autores obtiveram $69 \%$ de taxas de complicações que incluíam exposição da placa e deformidade de contorno. Resultados que não corroboram com os encontrados por $\mathrm{Li}$ et al. ${ }^{15}$ ao descreverem o uso de retalhos de tecido mole e placas de reconstrução em 116 pacientes com defeitos mandibulares. Seus resultados foram excelentes em termos de sobrevivência de tecido mole, e os atributos de exposição ou falha foram inferiores a $10 \%$. Os autores consideram que os resultados cosméticos foram aceitáveis e bastante semelhantes aos da reconstrução com enxerto ósseo. Setenta por cento dos pacientes tiveram restauração da deglutição, fala e abertura bucal.

No presente caso clínico relatado o defeito ósseo se estendia do corpo a sínfise mandibular, além um apagamento do fundo de vestíbulo mandibular e uma camada delgada de mucosa recobrindo a placa de reconstrução, sem aparente recobrimento muscular e comprometimento da mobilidade da língua. A nova placa instalada foi totalmente coberta por tecido muscular em camadas e por mucosa oral e restabelecido os aspectos anatômicos e funcionais satisfatório; respeitando assim os princípios preconizados pela literatura $^{9,13}$.

\section{CONSIDERACÕES FINAIS}

Levando em consideração as características do paciente e o objetivo funcional e estético, algumas opções de reconstrutivas estão disponíveis para pacientes que apresentam defeitos mandibulares provenientes de sequela pós-ressecção de lesão óssea. $\mathrm{O}$ uso da técnica da prototipagem foi um fator importante no resultado final do caso relatado, alcançando boa simetria facial e função mandibular, além de diminuir o tempo cirúrgico.

Apesar de algumas classificações dos defeitos mandibulares vêm sendo discutido ao longo dos anos, nenhuma é universalmente usada como protocolo para reconstrução mandibular. Sendo assim o cirurgião, baseado na sua experiência e na literatura, tem autonomia para propor o melhor método de tratamento para reconstrução mandibular em cada caso.

\section{REFERÊNCIAS}

1. Santos LCS, Seixas AM, Barbosa B, Cincura RNS. Adaptação de placas reconstrutivas: uma nova técnica. Rev Cir Traumatol Buco-MaxiloFac. 2011;11(2):9-14.

2. Lin PY, Lin KC, Jeng SF. Oromandibular reconstruction: the history, operative options and strategies, and our experience. ISRN Surg. 2011;2011:824251.

3. Martins Jr. JC, Keim FS. Uso de prototipagem no planejamento de reconstrução microcirúrgica da mandíbula. Rev Bras Cir Craniomaxilofac. 2011;14(4):225-28.

4. Montoro JR, Tavares MG, Melo DH, Franco Rde L, Mello-Filho FV, Xavier SP, Trivellato AE, Lucas AS. Mandibular ameloblastoma treated by bone resection and imediate reconstruction. Braz $\mathbf{J}$ Otorhinolaryngol. 2008;74(1):155-57.

5. Nóia CF, Ortega-Lopes R, Chaves Netto HDM, Nascimento FFAO, Sá BCM. Desafios na reconstrução mandibular devido a lesões extensas ou traumatismos. Rev. Assoc. Paul. Cir. Dent. 2015;69(2):158-63.

6. Cohen A, Laviv A, Berman P, Nashef R, AbuTair J. Mandibular reconstruction using stereolithographic 3-dimensional printing modeling technology. Oral Surg Oral Med Oral Pathol Oral Radiol Endod. 2009;108(5):661-6.

7. Rana M, Warraich R, Kokemüller H, Lemound J, Essig H, Tavassol $\mathrm{F}$ et al. Reconstruction of mandibular defects - clinical retrospective research over a 10-year period. Head Neck Oncol. 2011;3:23.

8. Fariña R, Alister JP, Uribe F, Olate S, Arriagada A. Indications of Free Grafts in Mandibular Reconstruction, after Removing Benign Tumors: Treatment Algorithm. Plast Reconstr Surg Glob Open. 2016;4(8):e845. 
9. Fariña R, Plaza C, Martinovic G. New transference technique of position of mandibular reconstructing plates using stereolithographic models. J Oral Maxillofac Surg. 2009;7(11):254448.

10. Mooren RE, Merkx MA, Kessler PA, Jansen JA, Stoelinga PJ. Reconstruction of the mandible using preshaped $2.3-\mathrm{mm}$ titanium plates, autogenous cortical bone plates, particulate cancellous bone, and platelet-rich plasma: a retrospective analysis of 20 patients. J Oral Maxillofac Surg. 2010;68(10):2459-67.

11. Brown JS, Barry C, Ho M, Shaw R.A new classification for mandibular defects after oncological resection. Lancet Oncol. 2016; 17(1):23-30

12.Urken ML, Weinberg H, Vickery $\mathrm{C}$, Buchbinder D, Lawson W, Biller HF. Oromandibular reconstruction using microvascular composite free flaps. Report of 71 cases and a new classification scheme for bony, soft-tissue, and neurologic defects. Arch Otolaryngol Head Neck Surg. 1991;117(7):733-44.

13. Shnayder Y, Lin D, Desai SC, Nussenbaum B, Sand JP, Wax MK. Reconstruction of the Lateral Mandibular Defect: A Review and Treatment Algorithm. JAMA Facial Plast Surg. 2015;17(5):367-73.

14. Wei FC, Celik N, YangWG, Chen IH, Chang YM, Chen HC. Complications after reconstruction by plate and soft-tissue free flap in composite mandibular defects and secondary salvage reconstruction with osteocutaneous flap. Plast Reconstr Surg. 2003;112(1):37-42.

15.Li BH, Jung HJ, Choi SW, Kim SM, Kim MJ, Lee JH. Latissimus dorsi (LD) free flap and reconstruction plate used for extensive maxillomandibular reconstruction after tumour ablation. $\mathbf{J}$ Craniomaxillofac Surg. 2012;40(8):293-300.
CONFLITO DE INTERESSES

Os autores declaram não haver conflitos de interesse.

AUTOR PARA CORRESPONDENCIA

Allancardi dos Santos Siqueira

allancardi@hotmail.com

Submetido em 19/02/2019

Aceito em 24/05/2019 